\title{
Routing of Military Communication Networks Based on Cloud Genetic Algorithm
}

\author{
Lei Ming ${ }^{1,2}$, Wang Heng ${ }^{1,2}$, Mao Shao-jie ${ }^{1,2}$, Yi Kan ${ }^{1,2}$ and Zhang Jin-feng ${ }^{1,2}$ \\ ${ }^{1}$ Science and Technology on Information Systems Engineering Laboratory, \\ Nanjing, 210007, Jiangsu, China \\ ${ }^{2}$ Collaborative Innovation Center of Novel Software Technology and \\ Industrialization, Nanjing, 210007, China \\ storm_sky001@126.com
}

\begin{abstract}
This paper aims at practical application needs of information transmission in existing military communication networks, and proposes routing models of military communication networks that satisfy military operational requirements. As routing is $N P$-hard problem, it has problems such as easily being early-maturing and caught in local optimum if traditional genetic algorithms are used to solve this kind of problem. Targeting at problems above, a CGA algorithm for solution is designed, which is based on excellent characteristics of cloud model and integrates with basic principles of genetic algorithm. On the basis of cloud model, CGA algorithm can dynamically adjust the crossover probability and mutation probability according to its fitness value, which shows self-adaptive characteristics of genetic parameters and reflect randomness and uncertainty during natural search process; thus the method is with good tendency and can promote diversity of population, and it not only improves the search efficiency, speeds up convergence, but also prevents algorithm from local optimum. The algorithm is then applied to route selection of the military communication network in a C4ISR simulation system. Experimental results show that the algorithm features a better convergence, and that although the run time of CGA algorithm is longer than that of AGA, the increment rate is within the tolerance interval since it does not exceed $10 \%$.
\end{abstract}

Keywords: military communication networks, routing, genetic algorithm, cloud model, crossover operator; mutation operator

\section{Introduction}

A military communication network, which is mainly used for military information transmission, is composed of various communication equipment, facilities, and related network management/control equipment, and is established according to certain organizational structure and connection method [1]. Under the warfare background based on information and high technology, a military communication network is regarded as a carrier of information, and a link connecting various operational units, and plays a critical and pivotal role.

The routing of military communication networks is considered as a critical technology in ensuring quick, accurate and safe and reliable information transmission, and an important issue in military communication networks design and operation. This paper studies the optimization of routing in military communication networks, and focuses on how to select scientific and rational communication routes by utilizing existing communication resources in military communication networks, thus to transmit correct information to needed nodes through the rational routes, avoid information overload, information transmission failure or serious delay in a link, and ensure smooth and 
efficient information transmission throughout the whole military communication network. The paper considers characteristics of several optimal objects including high timeliness and robustness in routing of military communication networks, establishes a multi-objective and multi-constrained optimized mathematical model for solving routing problems of military communication networks, and adopts cloud genetic algorithm as a special intelligent optimization algorithm to optimize the model solution.

\section{Routing Model of Military Communication Networks}

\subsection{Description of Problem and Parameter Calibration}

The routing metrics of military communication networks mainly include cost, hop, bandwidth, reliability, delay, jitter etc. Routing is for selecting one or more routes that have optimized these metrics. In order to avoid over-long or over-narrow routes, the paper takes the maximum hop and minimum bandwidth as constraints. Since delay and jitter can be fixed by bandwidths and hops collected, delay and jitter can be mapped to bandwidth constraints and hop constraints. To avoid certain communication node failures, which results in significant impact on information transmission of the entire communication network, the source number of information flow (source number of input information) transmitting through a certain node is taken as constraints. In order to consume less network resources and distribute load on idle link resources as far as possible so as to prevent network congestion and improve the throughput of network resources, the paper aims to optimize load balancing. The purpose of routing is to find transmission route of high efficiency under the premise that bandwidth and delay are ensured, by not only considering network topology, but also analyzing needs of data transmission and availability of link resources, and the problem is about optimization of multi-objective and multi-constrained model.

Assuming that a military communication network $G=(V, E), V$ is a set of communication nodes, $E$ is a set of communication links, $e \in E$ is a link of communication network G, symbols are defined as follows:

$R$ : a set of start communication nodes generating information flow, $R \subset V$;

$S$ : a set of destination communication nodes receiving information flow, $S \subset V$;

$|E|$ : the number of communication links;

$r$ : represents a start communication node, $r \in R$;

$s:$ indicates a destination communication node, $s \in S$;

$V_{\mathrm{rs}}$ : the set of all routes that connect communication node pair $r$-s from start to destination,

$r \in R, \quad s \in S$;

$q_{r s}$ : information flow sent from $r$ to $s$ during the study period, $r \in R, s \in S$;

$h_{v}^{r s}$ : information flow of the $v^{\text {th }}$ route between start-destination communication node pair $r-s, v \in V_{r s}, r \in R, s \in S$;

$x_{e}$ : workload on link $e, e \in E$;

$u_{e}$ : effective load capacity on link $e, e \in E$;

$c_{e}$ : delay on the link $e, e \in E$;

$b(e)$ : bandwidth on link $e, e \in E$;

$p_{v}^{r s}$ : hop of the $v^{\text {th }}$ route between start-destination communication node pair $r-s, r \in R$, $s \in S$; 
$\delta_{e, v}^{r s}=\left\{\begin{array}{l}1, \text { If link e is located at the } \mathrm{v}^{\text {th }} \text { route of communication node pair r-s that } \\ \text { connects start-destination } \\ 0, \text { Others }\end{array}\right.$

\subsection{Constraints of Problems}

\subsubsection{Constraints from Hops of Information Transmission Routes}

If an information transmission route contains excessive hops in a military communication network, so that the information transmission will be delayed and the transmission quality will be degraded, it is undoubted that such phenomenon is not consistent with timeliness of military communication networks. The paper assumes that all hops of information transmission routes throughout the military communication network must meet certain constraints, and assumes $\sigma_{\max }$ is the maximum hops of all information transmission routes.

The actual number of hops in information transmission route is $p_{v}^{r s}, p_{v}^{r s}$ must be less or equal to $\sigma_{\max }$ :

$$
p_{v}^{r s} \leq \sigma_{\max }
$$

\subsubsection{Constraints from Delay of Information Transmission Routes}

In military communication networks, different nodes require different information transmission delay, which depends on the importance of the nodes and information, and urgency of the task, etc.. The delay can be obtained by expert scoring method, and thus is not described herein.

Assuming that the actual transmission delay of information transmission route $v \in V_{r s}$ is $t_{v}^{r s}, t_{v}^{r s}$ must be less than or equal to required delay constraint $T_{r-s}$, as follows:

$$
t_{v}^{r s} \leq T_{r s}
$$

\subsubsection{Constraints from Number of Traffic Sources of Information Transmission Routes}

The routing transmission of military communication networks must have a certain invulnerability, so that when a certain communication node fails in military communication networks, the entire information transmitted over the military networks will not be completely lost,; the number of information sources (i.e. number of information sources passing through this communication node) passing through certain communication node should not be too large, otherwise, the entire communication networks will be significantly impacted if this node fails.

$$
\sum_{v} \sum_{r, s} \delta_{e, v}^{r s} \leq Q(e \in E)
$$

where $Q$ is the source number of information flow communicated on the link.

\subsubsection{Constraints from Bandwidth of Information Transmission Routes}

The bandwidth available on a route is equal to the minimum of link bandwidth on route, in order to avoid too narrow route, assuming that

$$
\min \left(b_{v}^{r s}\right) \geq B \quad y \in V_{r s},
$$

where $B$ is the minimum of link bandwidth. 


\subsection{Objective Function of the Problem}

The objective function aims to not only satisfy the constraints on communication networks and information exchange between communication networks nodes, minimize average delay of information transmission in the entire military communication networks, but also balance network load by optimizing information transmission routing.

Information transmission delay is calculated as follow:

$$
T(x)=\sum_{e \in E} c_{e}
$$

Root-mean-square of network load is:

$$
S(x)=\sqrt{\frac{1}{|E|} \sum_{e \in E}\left(\frac{x_{e}}{u_{e}}-\frac{1}{|E|} \sum_{e \in E} \frac{x_{e}}{u_{e}}\right)^{2}}
$$

\section{4t Mathematical Model of the Problem}

To facilitate explanation, this paper takes information transmission delay, network load as the target criteria, thus to minimize routing transmission delay and RMS of the loads. Taking routing hops, delay, traffic constraints, and minimum bandwidth as the constraint conditions, the mathematical model of optimal routing in entire military communication networks is described below,

$$
\begin{aligned}
& \min F(x)=(T(x), S(x)) \\
& \text { thereinto, } T(x)=\sum_{e \in E} c_{e} \\
& S(x)=\sqrt{\frac{1}{|E|} \sum_{e \in E}\left(\frac{x_{e}}{u_{e}}-\frac{1}{|E|} \sum_{e \in E} \frac{x_{e}}{u_{e}}\right)^{2}} \\
& \text { s.t. }\left\{\begin{array}{l}
h_{v}^{r s}=0 \text { 或 } h_{v}^{r s}=q_{r s}, \forall r \in R, s \in S, v \in V_{r s} \\
x_{e}=\sum_{r} \sum_{s} \sum_{v} h_{v}^{r s} \delta_{e, v}^{r s} \leq u_{e}, \forall e \in E, \forall r \in R, s \in S, v \in V_{r s} \\
p_{v}^{r s} \leq \sigma_{\max }, \forall r \in R, s \in S, v \in V_{r s}, \forall r \in R, s \in S, v \in V_{r s} \\
\sum_{v} \sum_{r, s} \delta_{e, v}^{r s} \leq Q, \forall e \in E, \forall r \in R, s \in S, v \in V_{r s} \\
\min _{e}\left(b_{v}^{r s}\right) \geq B, \forall r \in R, s \in S, v \in V_{r s}
\end{array}\right.
\end{aligned}
$$

Military communication networks contain many different types of routing parameters, the main idea is to consider them as objective functions and constraints. Therefore, the model can be enlarged to accommodate any objective function and constraint. It should be noted that the route optimization contains different dimensions and properties, and it must be normalized and standardized first. It is not the main subject of the paper, and thus isn't described here $[2,3]$.

\section{Problem Solving}

As a kind of combinational optimization problem, routing of military communication networks has been proven to be a NP-hard problem [4]. Although traditional approaches including gradient method and dynamic programming can be used to solve it, these methods have various limits, especially for large-scale network. Heuristic algorithms or intelligent algorithms such as genetic algorithm [5], tabu search [6] and ant colony 
algorithm [7] are often adopted as a solution, but they are easy to be caught in local optimum and premature convergence when solving routing.

Proposed by Professor John Holland from Michigan University in the United States in 1975, Genetic Algorithm (GA) is a heuristic global optimization algorithm, which simulates biological population genetics and evolutionary processes and is characterized by self-adaptive ability, parallelism, good global optimization and Robustness [8,9]. In theory, genetic algorithm has the capacity to find the global optimal solution, but it also has some disadvantages, such as being early-maturing and local optimum. Standard genetic algorithm (SGA) adopts fixed probability of crossover and mutation, and suffers a series of disadvantages such as the tendency to be caught in local optimum and slow convergence; it is required to do a lot of experiments to set better genetic parameters for specific problems, and this brings inconvenience to problem solving $[10,11,12]$.

How to better reflect the tendency and randomness of the genetic evolution process is a key issue of genetic algorithm research. Fuzzy theory is introduced $[13,14,15]$, which can reflect the tendency and randomness of natural evolutionary process well. However, owing to the limits of fuzzy set theory, there will not be fuzziness when the membership function is considered as the assumed one. Generally speaking, natural language is more suitable for describing the adaptiveness of genetic parameters. First proposed by Academician Li DY in 1995, the cloud model is an uncertainty transformation model between the language-expressed concept and its quantitative representation, and can be used for reflecting the uncertainty of natural language concepts. Characterized by both randomness and ambiguity, it provides a powerful way of conversion between qualitative and quantitative concepts, and can be used to present abundant uncertainty phenomenon. Over ten years of improvement and development, the cloud model has been successfully applied in intelligent control, data mining, evaluation, knowledge representation, algorithm improvements and other fields $[14,15,16]$. Based on fundamental principles of genetic algorithm and excellent characteristics of cloud model, this paper proposes a route selection algorithm (CGA). This algorithm generates crossover operator and mutation operator through cloud model. Meanwhile, the preservation strategy of the optimal individual and the protection strategy of the worst individual are adopted for improving genetic operation, and thus the search efficiency of algorithm and convergence rate can be enhanced, and the likelihood of getting caught in the local optimum can be avoided. Consequently, the performance of the algorithm will be observably improved.

\subsection{Cloud Model Theory}

Definition 1

Assuming $U$ is a quantitative domain represented with precise values (one-dimensional, two-dimensional or multi-dimensional), and $\tilde{A}$ is a qualitative concept on the domain $U$. If there exists a random number $\mu(x) \in[0,1]$ with stable tendency from any element $x$ in $U$

$$
\mu: U \rightarrow[0,1], \forall x \in U, x \rightarrow \mu(x)
$$

the qualitative concept $\tilde{A}$ mapping from the domain $U$ to the interval [0,1] is distribution of $x$ in domain $U$, called as $\tilde{A}$ cloud model, referred to as cloud [16]. Each $x$ is called as a cloud drop, $\mu(x)$ is called as degree of certainty of $x$ on $\tilde{A}$. Random quantitative value $x$ represents randomness of quantitative values as the concept, but $\mu(x)$, the degree of certainty of $x$ on $\tilde{A}$ shows the extent of certainty where the value $x$ represents qualitative concepts.

Cloud takes expectation Ex, entropy En and hyper entropy $H e$ as three numerical eigenvalues to characterize a concept [16]. Such three numerical eigenvalues of cloud model fully integrates fuzziness and randomness by constructing specified algorithm, thus to form a conversion model between qualitative concept and quantitative representation. Cloud model doesn't emphasize precise function representation any longer, but shows the 
concept uncertainty by three numerical eigenvalues, represents uncertainty conversion between qualitative concept and quantitative representation by specific algorithms, and reveals relevance between fuzziness and randomness.

Expectation Ex is an expectation on spatial distribution of cloud drops in domain, namely the point in domain space that is capable of representing qualitative concept. It can represent excellent characteristics of parent individual heredity, and it is inherited from parent by offspring. Entropy En reflects the concept's randomness and fuzziness. Hyper entropy $H e$ is uncertainty value of En, namely entropy of the entropy, which is determined by the randomness and fuzziness of entropy. En and He can represent the uncertainty and fuzziness of the inheritance process, reflecting the variation features of species' evolutionary process.

Cloud model is a transformation model between qualitative knowledge and quantitative data. It can present qualitative knowledge via quantitative data, namely the process of generating cloud drops through numerical characteristics, which can be referred to as a forward cloud generator; conversely, the quantitative data can present the process of generating qualitative concept, namely the process of obtaining the numerical characteristics of cloud model through the cloud drop distribution, which we call it backward cloud generator [16].

Assuming that $E X 1=30, E n 1=2, H e 1=0.1$ and $E X 2=30, E n 2=5, H e 2=1, n=1000$, one-dimensional normal cloud drawing of joint distribution $\mathrm{C}(30,2,0.1)$ and $\mathrm{C}(30,5,0.3)$ respectively generated is as shown in Figure 1, the concept in Figure 1 is "in the vicinity of 30", entropy and hyper entropy can quantify such a qualitative concept. Cloud drops in Figure 1 can be understood as two populations with 1000 individuals obtained by different entropy and hyper entropy. Two populations are significantly different in coverage and dispersion degree: the greater the entropy is, the greater the coverage of individual will be; the greater the hyper entropy is, the more disperse the individuals will be. In this paper, the genetic operators are expressed by cloud models' three numerical eigenvalues, especially, $\mathrm{He}$ is capable of effectively representing stable tendency and randomness of evolution: the less $\mathrm{He}$ is, the weaker the uncertainty will be; the greater $\mathrm{He}$ is, the stronger the uncertainty will be.
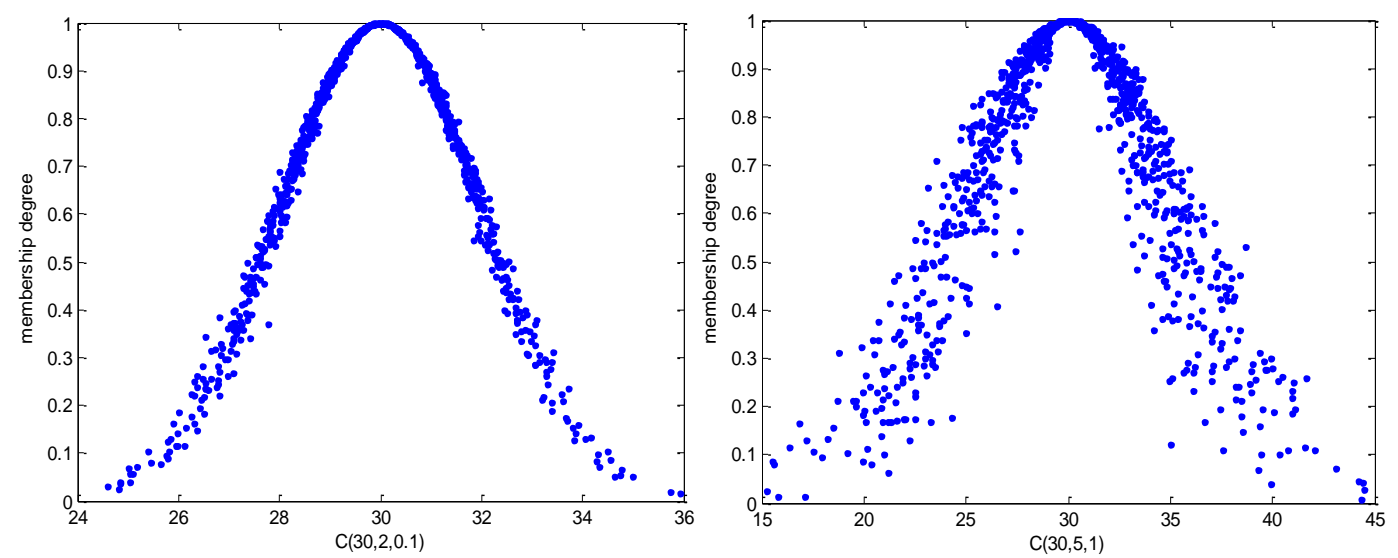

Figure 1. Conceptual Expression of Cloud Model: "in the Vicinity of 30"

\subsection{Encoding Method of Chromosome}

In genetic algorithm, encoding is the first step of design. Generally, binary encoding is used for individuals. When the network is large, encoding scheme will make solution space of the problem very large so that search efficiency is low. Military communication networks that are researched in this essay is not wholly connected and plenty of 
disconnected links exist. Therefore, construction of integer encoding based on route is needed. According to topological structure of network, candidate route set of links will be generated first. Element combinations of this set can form the solution space of routing of military communication networks.

Set the number of different information transmission within the military communication network is $N(N \leq D \cdot(D-1, D=|E|$ is node number of the network). Suppose information transmission from $V_{m}$ (the communication node generating information flow) to $V_{n}$ (the communication node receiving information flow) is the $d^{\text {th }}$ $(d=1,2, \ldots, N)$ information transmission among $N$, and the set of all possible information transmission routes of the $\mathrm{d}^{\text {th }}$ information transmission is Path ${ }_{m \rightarrow n}$ which satisfies constraint condition(8)-(13). Let $\Omega_{p_{d}}=\left\{p_{d}^{1}, \ldots, p_{d}^{k}, \ldots, p_{d}^{r_{d}}\right\}, m$ and $n$ are natural numbers, $V_{m} \in R, V_{n} \in S$, and $r_{d}$ refers to the number of all possible information transmission routes of the $\mathrm{d}^{\text {th }}$ information transmission, and $p_{d}^{k}\left(k=1,2, \ldots, r_{d}\right)$ represents the $k^{\text {th }}$ information transmission route among all possible information transmission routes. An information transmission route combination is composed of any one of all information transmissions, which is $P C=\left\{\left(p_{1}, \ldots, p_{d}, \ldots, p_{N}\right) \mid p_{d} \in \Omega_{P_{d}}, d=1,2, \ldots, N\right\}$. When $D=5$, suppose all units have the requirement for information transmission and they all meet the constraint condition (4)-(8), then $N=D \cdot(D-1)=20, \quad r_{d}=4(d=1,2, \ldots, 20)$. Suppose information transmission from communication node 1 to node 2 is the $1^{\text {st }}$ information transmission of the military communication network, then all information transmission routes of this $1^{\text {st }}$ information transmission can be defined as: $p_{1}^{1}$ corresponds to $1 \rightarrow 2$, $p_{1}^{2}$ corresponds to $1 \rightarrow 3 \rightarrow 5 \rightarrow 2, \quad p_{1}^{3}$ corresponds to $1 \rightarrow 3 \rightarrow 5 \rightarrow 4 \rightarrow 2, \quad p_{1}^{4}$ corresponds to $1 \rightarrow 5 \rightarrow 2$ and $p_{1}^{5}$ corresponds to $1 \rightarrow 5 \rightarrow 4 \rightarrow 2$ (See Figure 2 and Table 1.)

A chromosome corresponds to a certain information transmission route combination. Therefore, integer encoding can be adopted to encode chromosome of CGA: a chromosome is an ordered sequence $\boldsymbol{\omega}=\left\{\omega_{1}, \ldots, \omega_{d}, \ldots, \omega_{N}\right\}$ composed of $N$ integers. Hereinto, $\omega_{d} \in\left\{1,2, \ldots, r_{d}\right\}, d=1,2, \ldots, N$. If $\omega_{d}=k$, it represents the $k^{\text {th }}$ information transmission route in $\Omega_{P_{d}}$ is selected (See Figure 3.)

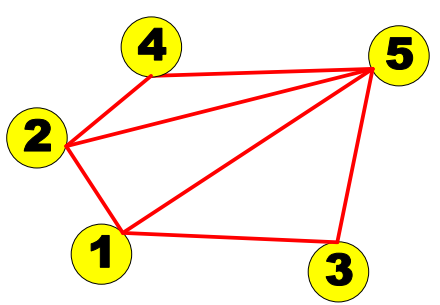

Figure 2. Sketch Map of a Communication Network

\section{Table 1. Set of Candidate Routes Generated By Algorithm (Hop Count Is Set Not More than 3)}

\begin{tabular}{|c|c|c|c|}
\hline No. & Information transmission & Information transmission route & Code \\
\hline \multirow{3}{*}{1} & \multirow{3}{*}{$1 \rightarrow 2$} & $1 \rightarrow 2$ & 1 \\
\cline { 3 - 4 } & \multirow{3}{*}{$1 \rightarrow 2$} & $1 \rightarrow 3 \rightarrow 5 \rightarrow 2$ & 2 \\
\cline { 3 - 4 } & & $1 \rightarrow 5 \rightarrow 2$ & 3 \\
\hline
\end{tabular}




\begin{tabular}{|c|c|c|c|}
\hline \multirow{3}{*}{2} & \multirow{3}{*}{$1 \rightarrow 3$} & $1 \rightarrow 2 \rightarrow 5 \rightarrow 3$ & 1 \\
\hline & & $1 \rightarrow 3$ & 2 \\
\hline & & $1 \rightarrow 5 \rightarrow 3$ & 3 \\
\hline \multirow{5}{*}{3} & \multirow{5}{*}{$1 \rightarrow 4$} & $1 \rightarrow 2 \rightarrow 4$ & 1 \\
\hline & & $1 \rightarrow 2 \rightarrow 5 \rightarrow 4$ & 2 \\
\hline & & $1 \rightarrow 3 \rightarrow 5 \rightarrow 4$ & 3 \\
\hline & & $1 \rightarrow 5 \rightarrow 2 \rightarrow 4$ & 4 \\
\hline & & $1 \rightarrow 5 \rightarrow 4$ & 5 \\
\hline \multirow{4}{*}{4} & \multirow{4}{*}{$1 \rightarrow 5$} & $1 \rightarrow 2 \rightarrow 4 \rightarrow 5$ & 1 \\
\hline & & $1 \rightarrow 2 \rightarrow 5$ & 2 \\
\hline & & $1 \rightarrow 3 \rightarrow 5$ & 3 \\
\hline & & $1 \rightarrow 5$ & 4 \\
\hline \multirow{4}{*}{5} & \multirow{4}{*}{$2 \rightarrow 1$} & $2 \rightarrow 1$ & 1 \\
\hline & & $2 \rightarrow 4 \rightarrow 5 \rightarrow 1$ & 2 \\
\hline & & $2 \rightarrow 5 \rightarrow 1$ & 3 \\
\hline & & $2 \rightarrow 5 \rightarrow 3 \rightarrow 1$ & 4 \\
\hline$\ldots$ & $\ldots$ & $\ldots$ & 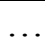 \\
\hline
\end{tabular}

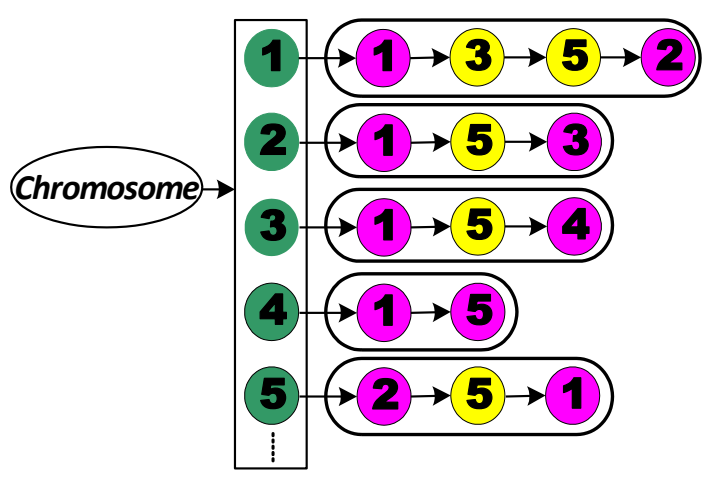

Figure 3. Sketch Map of Chromosome Encoding

\subsection{Fitness Function}

It can be concluded from the above chromosome encoding method that infeasible individuals may exist in the obtained chromosomes (it means the chromosomes that do not meet constraint condition of the military communication network.) Therefore, before calculating fitness value of chromosome, feasibility of all chromosomes needs to be judged to see if it meets the constraints. If not, fitness value of this chromosome shall be punished and fitness value of this chromosome will be set as 0 directly. At the same time, according to the above model, the problem is multi-target and multi-constraint, and the extreme point of all objective functions may not always exist. Therefore, fitness function constructed in this paper is

$$
\text { Fitness }=\frac{2}{e^{T}\left(1+e^{S}\right)}
$$

In short, fitness value is in $[0,1]$ and

$$
\text { Fitness }= \begin{cases}\frac{1}{e^{T}\left(1+e^{S}\right)}, & \text { the individual meets the constraint conditions } \\ 0, & \text { else }\end{cases}
$$




\subsection{Genetic Manipulation}

\subsubsection{Generation of Initial Population}

According to the chromosome coding process, an information transmission route combination is formed by any information transmission route combination among all information transmission routes, and it is recorded as $P C=\left\{\left(p_{1}, \ldots, p_{d}, \ldots, p_{N}\right) \mid p_{d} \in\right.$ $\left.\Omega_{P_{d}}, d=1,2, \ldots, N\right\} . \Omega_{p_{d}}=\left\{p_{d}^{1}, \ldots, p_{d}^{k}, \ldots, p_{d}^{r_{d}}\right\}, r_{d}$ represents the number of all possible information transmission routes of the $\mathrm{d}^{\text {th }}$ information transmission, and $p_{d}^{k}\left(k=1,2, \ldots, r_{d}\right)$ represents the $\mathrm{k}^{\text {th }}$ information transmission route of all possible information transmission routes. A chromosome is an ordered sequence $\omega=\left\{\omega_{1}, \ldots, \omega_{d}, \ldots, \omega_{N}\right\}$ formed by $\mathrm{N}$ integers, and $\omega_{d} \in\left\{1,2, \ldots, r_{d}\right\}, \quad d=1,2, \ldots, N$.

The process of generating population that includes $U$ chromosomes is: to generate $\mathrm{N}$ random number respectively, record them as $\omega_{11}, \ldots, \omega_{d l}, \ldots, \omega_{N l}$, where $\omega_{d l}$ is the integer random number from 1 to $r_{d}$, namely $\omega_{d l} \in\left\{1,2, \ldots, r_{d}\right\}, d=1,2, \ldots, N$. Therefore, a chromosome is obtained, and it is recorded as $\omega_{1}=\left\{\omega_{11}, \ldots, \omega_{d 1}, \ldots, \omega_{N 1}\right\}$. This process is repeated $U$ times, and $U$ chromosomes are obtained, and are recorded as $\Omega_{\omega}=\left\{\omega_{1}, \ldots, \omega_{k}, \ldots, \omega_{U}\right\}$, wherein $\omega_{k}=\left\{\omega_{1 k}, \ldots, \omega_{d k}, \ldots, \omega_{N k}\right\}, \omega_{d k} \in\left\{1,2, \ldots, r_{d}\right\}, d=1,2, \ldots, N$, $k=1,2, \ldots, U$.

This group represents the set of some possible solutions of route selection for the military communication network. Generally, they are not good, and what needs doing is to simulate the evolutionary process on the basis of these groups, to select the superior and eliminate the inferior ones in order to get excellent groups and individuals.

\subsubsection{Selection Operator}

In basic genetic algorithm, probability that individual may be copied is determined by its fitness value. As this is random, the best individual may be lost during search. In order to ensure the final result obtained when CGA ends is the chromosome with highest fitness value in all generations and to improve operating efficiency and convergence of algorithm at the same time, preservation percentage $P_{\text {save }}$ is set, i.e. to copy the best individual with probability of $P_{\text {save }}$ to next generation directly. At the same time, in order to protect population diversity, preservation probability $P_{p r o}$ is set, and with the $P_{p r o}$ the worst individual of the lowest fitness in population is inherited to next generation.

\subsubsection{Crossover Operator and Mutation Operator}

Crossover probability algorithm of self adaption based on cloud is as follow:

$$
\begin{aligned}
& E X=\frac{f_{1}+f_{2}}{2} \\
& E n=l_{1}\left(f_{\max }-f^{\prime}\right) \\
& H e=l_{2} E n \\
& E n=\operatorname{Norm}(E n, H e) \\
& P_{c}^{\prime}= \begin{cases}k_{1} e^{\frac{-\left(f^{\prime}-E x\right)^{2}}{2(E n)^{2}}}, f^{\prime} \geq \bar{f} \\
k_{2}, & f^{\prime}<\bar{f}\end{cases}
\end{aligned}
$$

where $P_{c}^{\prime}$ refers to crossover probability, $f_{\max }$ is the maximum fitness value of population, $f_{\text {ave }}$ is the average fitness, $f_{1}$ and $f_{2}$ respectively refer to fitness value of two 
crossed individuals, $f^{\prime}$ is the greater fitness value of two crossed individuals,

$l_{1} 、 l_{2} 、 k_{1} 、 k_{2}$ refer to control parameters, and

$l_{1} \in[0.3,0.5], l_{2} \in[0.5,0.7] 、 k_{1}, k_{2} \in[0,1]$. Let $l_{1}=l_{2}=0.5$, and $k_{1}=k_{2}=1$.

Mutation probability algorithm of self adaption based on cloud is as follow:

$$
\begin{aligned}
& E X=f \\
& E n=l_{3}\left(f_{\mathrm{m} \mathrm{a}} \bar{x} f\right) \\
& H e=l_{4} E n \\
& E n^{\prime}=\operatorname{Norm}(E n, H e) \\
& P_{m}^{\prime}= \begin{cases}k_{3} e^{\frac{-(f-E x)^{2}}{2(E n)^{2}}}, f \geq \bar{f} \\
k_{4}, & f<\bar{f}\end{cases}
\end{aligned}
$$

where $P_{m}^{\prime}$ refers to mutation probability, $f_{\max }$ is the maximum fitness value of population, $f$ is the fitness value of mutational individual, and $l_{3}, l_{4}, k_{3}, k_{4}$ are control parameters. Thereinto, $l_{3} \in[0.05,0.2] 、 l_{4} \in[0.05,0.25], k_{3}, k_{4} \in[0,1]$. Let $l_{3}=l_{4}=0.1$,

$k_{1}=k_{2}=0.5$.

As a result, for individuals having fitness value higher than average in the population, CGA algorithm will ensure that the higher the fitness value is, the lower the probability of crossover and mutation becomes with the value being higher than 0 , so as to "protect" the excellent individuals; but for those individuals with lower fitness value, the algorithm will adopt higher crossover mutation probability to generate superior individuals.

In order to ensure legality of chromosome, mutation point shall be mutated in candidate route, which will make chromosome choose mutated route in feasible route candidate set. In this way, there is no need to conduct coding test, so as to greatly improve arithmetic speed.

\subsubsection{Termination Condition for CGA Operation}

Routing is a NP-hard problem. Genetic algorithm gets close to optimal solution through successive iteration. As lower limit value of objective function is hard to confirm, the method of setting maximum number of iterations simply is not applicable. Maximum number of iterations have been set during design, and when the fitness value of an individual does not change or change little within the maximum number of iterations, convergence of the group will be judged accordingly and iteration process is finished. Or else, iteration will be continued. After iteration stops, the individual with highest fitness value will be chosen as optimal scheme, and the routing scheme will be obtained after the individual is decoded.

\subsection{Arithmetic Steps}

Flow chart of CGA is showed as Figure 4. 


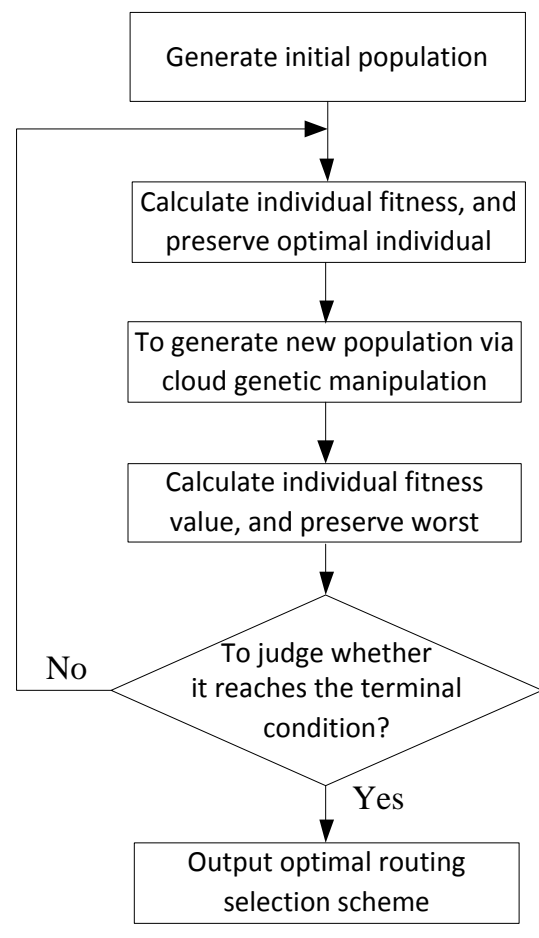

Figure 4. Flow Chart of CGA

\section{Simulation Verification}

In this paper, the military communication network simulation system, which is part of a united air defense C4ISR system performance evaluation platform, is developed with VC 8.0. According to Kleinrock Queuing Theory, suppose information on each link in information interactive network is based on $\mathrm{M} / \mathrm{M} / 1$ queue model and suppose information transmission processes on each link do not influence each other [17]. Total number of various nodes in this example is 58 . Hereinto, there are 20 communication nodes, 3 information processing nodes, 15 detection nodes, 14 weapon platform user nodes and 6 control nodes. Simplified network structure is shown in Figure 5. Simulation verification is conducted with data transmission of the military communication network in 6 time periods in a simulation experiment. 


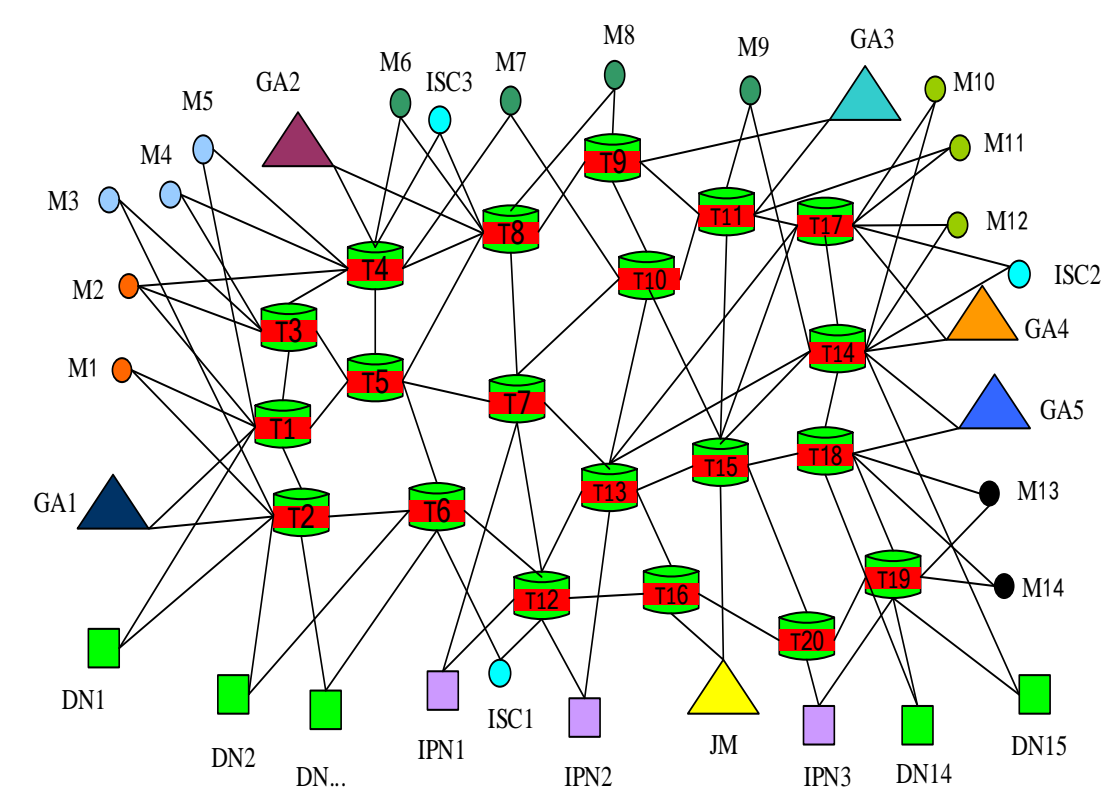

\section{Figure 5. Network Topology of the Military Communication Network in a Simulation Experiment}

Where DN refers to Detection Node, IPN refers to Information Processing Node, ISC refers to Information Service Center, GA refers to Group Army, M refers to Military, and JM refers to Joint Command.

\subsection{Experiment on Algorithm Convergence}

In order to analyze the convergence of the algorithm, independent experiments are conducted for 50 times on data transmission of the military communication network in 6 time periods with algorithm. The main parameters of the algorithm include: the population size being 300 and the maximum iteration number being 100. The experimental result is as shown in Figure 6. Hereinto, the ordinate refers to the average value of optimal values that are searched in this generation in 30 experiments with algorithm. From Figure 6, it can be concluded that calculation for the 6 time periods can be converged quickly. With iterations increase, fitness value self-adaptively evolves towards optimal direction. Calculation of all time periods can reach or get very close to optimal solution in the first 50 generations. The algorithm shows a better convergence, which is able to reduce time complexity obviously.

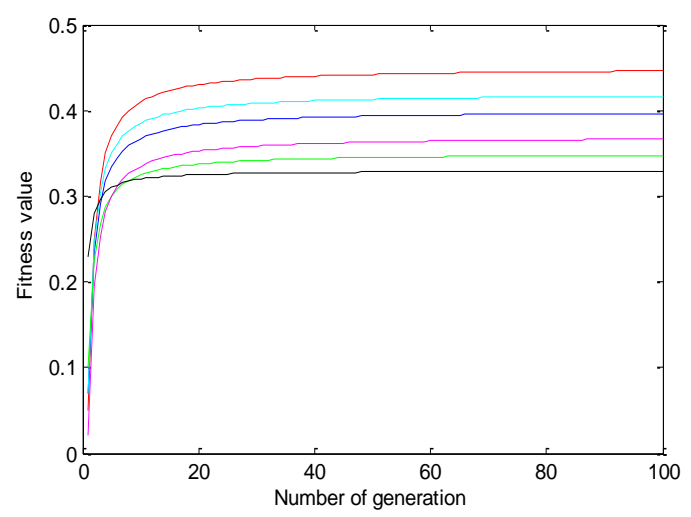

Figure 6. Convergence Curve of 6 Time Periods 


\subsection{Analysis on Contrast Experiment of Global Searching Ability of Algorithm}

In order to analyze global searching ability of the algorithm, Comparison have been made between the algorithm with AGA and SGA separately on 6 time periods. Parameter setting here is the same as in the convergence experiment. Experimental results are shown as Table 2.

Table 2. Comparison of Algorithm Performance

\begin{tabular}{|c|c|c|c|c|c|}
\hline $\begin{array}{l}\text { Simulation time } \\
\text { period of the }\end{array}$ & $\begin{array}{l}\text { Optimal } \\
\text { value }\end{array}$ & $\begin{array}{l}\text { Average } \\
\text { value }\end{array}$ & $\begin{array}{l}\text { Standard } \\
\text { deviation }\end{array}$ & $\begin{array}{l}\text { Unsuccessful } \\
\text { convergence }\end{array}$ & $\begin{array}{l}\text { Average } \\
\text { time }(\mathrm{s})\end{array}$ \\
\hline $\begin{array}{c}\text { military } \\
\text { communication } \\
\text { network }\end{array}$ & $\begin{array}{l}\text { CGA } \\
\text { SGA }\end{array}$ & $\begin{array}{l}\text { CGA } \\
\text { SGA }\end{array}$ & $\begin{array}{l}\text { CGA } \\
\text { SGA }\end{array}$ & $\begin{array}{l}\text { CGA } \\
\text { SGA }\end{array}$ & $\begin{array}{l}\text { CGA } \\
\text { SGA }\end{array}$ \\
\hline$T 1$ & $\begin{array}{l}0.651 \\
0.574\end{array}$ & $\begin{array}{l}0.623 \\
0.539\end{array}$ & $\begin{array}{c}0.0077 \\
0.083\end{array}$ & $\begin{array}{l}0.02 \\
0.11\end{array}$ & $\begin{array}{l}4.3 \\
4.4\end{array}$ \\
\hline$T 2$ & $\begin{array}{l}0.472 \\
0.441\end{array}$ & $\begin{array}{l}0.465 \\
0.393\end{array}$ & $\begin{array}{l}0.0021 \\
0.0395\end{array}$ & $\begin{array}{c}0 \\
0.09\end{array}$ & $\begin{array}{l}4.2 \\
4.4\end{array}$ \\
\hline$T 3$ & $\begin{array}{l}0.375 \\
0.345\end{array}$ & $\begin{array}{l}0.362 \\
0.322\end{array}$ & $\begin{array}{l}0.0027 \\
0.0363\end{array}$ & $\begin{array}{l}0.01 \\
0.09\end{array}$ & $\begin{array}{l}3.6 \\
3.7\end{array}$ \\
\hline$T 4$ & $\begin{array}{l}0.349 \\
0.312\end{array}$ & $\begin{array}{l}0.337 \\
0.317\end{array}$ & $\begin{array}{l}0.0011 \\
0.0189\end{array}$ & $\begin{array}{c}0 \\
0.12\end{array}$ & $\begin{array}{l}4.0 \\
4.2\end{array}$ \\
\hline$T 5$ & $\begin{array}{l}0.285 \\
0.273\end{array}$ & $\begin{array}{l}0.281 \\
0.265\end{array}$ & $\begin{array}{c}0.0003 \\
0.014\end{array}$ & $\begin{array}{c}0 \\
0.06\end{array}$ & $\begin{array}{l}2.8 \\
3.1\end{array}$ \\
\hline$T 6$ & $\begin{array}{l}0.236 \\
0.214\end{array}$ & $\begin{array}{l}0.229 \\
0.186\end{array}$ & 0.00070 .0130 & $\begin{array}{c}0 \\
0.05\end{array}$ & $\begin{array}{c}3.4 \\
3.5\end{array}$ \\
\hline
\end{tabular}

As is shown in Table 2, the performance of CGA and AGA is better than that of SGA. Owing to optimal target value, lower standard deviation, and least unsuccessful convergence, CGA is proved to be the best one with the strong capability of optimum searching. Standard deviation of the optimal solutions worked out by CGA is the lowest, and thus it has greater precision. Meanwhile, its lower unsuccessful convergence time proves that CGA algorithm is with good stability. As a result of the normal cloud adopted, the run time of CGA algorithm is longer than that of AGA, but the increment rate is within the tolerance interval since it does not exceed $10 \%$.

\section{Conclusions}

It is obvious that cloud genetic manipulation is capable to show randomness and uncertainty during natural search process, and reflect self-adaptive characteristics of genetic parameters. Therefore, the method is characterized by good tendency and promotion of the diversity of the population, and it not only improves the search efficiency, speeds up convergence, but also prevents the algorithm from being caught in local optimum. The algorithm is also applied to routing of the military communication network in a C4ISR simulation system, and experimental results show that the algorithm is feasible and effective.

\section{Acknowledgements}

This study was supported by Jiangsu 333 Leading Talents Cultivation Project (Program Number: BRA2014213) and Jiangsu Province Collaborative Innovation Center of Novel Software Technology and Industrialization. 


\section{References}

[1] "Military Terminology Management Committee of the PLA", the Military Academy; Military Terms of the PLA (full edition), Military Science Press, (2011).

[2] W. Chang, "Ranking of fuzzy utilities with triangular membership functions", Proceedings of International Conference on Policy Analysis and Systems, vol. 15, no.23, (1981).

[3] K. Atanassov, "Intuitionistic fuzzy sets Fuzzy Sets and Systems", Fuzzy Systems, vol. 20, no.1, (1986).

[4] W. Zheng and J. Crowerofl, "Quality of service routing for supporting multimedia applications", IEEE Journal on Selected Areas in Communications, vol 14, no.7, (1996).

[5] M. Munetomo, Y. Takai, Y. Sato, "An Adaptive Routing Algorithm with Load Balancing by a Genetic Algorithm", Trans. Of IPSJ, vol 399, no.2, (2008).

[6] J. Shen and F. Xu, "Study on optimization of capacity and flow distribution in computer communication network based on tabu search algorithm", Journal of Lanzhou University, vol. 39, no.3, (2003).

[7] S. Qi, Y. Li and D. Mei, "Application research on ant colony algorithm in QoS unicast routing", Journal of University of Electronic Science and Technology of China, vol 39, no.2, (2010).

[8] J. Chen, D. Guo and N. Xu, "Summary of theoretical studies on genetic algorithms", Journal of Xidian University, vol 25, no.3, (1998).

[9] Y. Lihua, "Theoretical research on genetic algorithm based on evolution of species", Nanjing University of Aeronautics and Astronautics Press, (2009).

[10] X. Wang and L. Cao, "Genetic algorithm theory, application and software implementation", Xi'an Jiaotong University Press, (2002).

[11] N. Song and S. Gao, "Genetic algorithm for solving multi-constraint QoS multicast routing problem", Computer Engineering, vol. 32, no. 24, (2006).

[12] T. Tang and W. Shen, "Wei Lingyun. QoS multicast routing optimization of DNA genetic algorithm". Computer Engineering, vol. 36, no.5, (2010).

[13] F. Herrera and M. Lozano, "Adaptation of genetic algorithm parameters based on fuzzy logic controller", Herrear F, Verdegay J1. Genetic Algorithms and soft Computing. Berlin Germany: Springer-Verla, (1996).

[14] D.Y. Li, "Uncertainty in knowledge representation", Chinese Engineering Science, vol. 2, no.10, (2000).

[15] D.Y. Li and C.Y. Liu, "Study on the universality of the normal cloud model", Chinese Engineering Science, vol. 6, no.8, (2004).

[16] D.Y. Li, Y. Du, "Artificial intelligence with Uncertainty", National Defense Industry Press, (2005).

[17] S. Fuli, "Research on modeling, analysis and reconstruction of military communications network based on super network", National University of Defense Technology, (2009). 